\title{
Fracionamento sequencial de cádmio e chumbo em solos
}

\author{
Sequential fractionation of cadmium and lead in soils
}

\author{
Claudia das Neves Costa ${ }^{\mathrm{I}}$ Egon José Meurer $^{\mathrm{II}}{ }^{*}$ Carlos Alberto Bissani ${ }^{\mathrm{II}}$ \\ Marino José Tedesco ${ }^{I I}$
}

\section{RESUMO}

\begin{abstract}
O impacto da contaminação e/ou poluição por metais pesados não deve ser avaliado somente pelo seu teor total em solos, mas pela sua biodisponibilidade, que é uma propriedade relacionada com sua mobilidade no solo e absorção pelas plantas. Este estudo teve por objetivo avaliar, mediante extrações químicas seqüenciais, a biodisponibilidade do cádmio e do chumbo em seis solos do Estado do Rio Grande do Sul. Para isto, utilizaram-se amostras nas quais foram adicionadas três doses de Cd (1,25, 2,50 e 5,0 $\mathrm{mg} \mathrm{kg}^{-1}$ de solo) e três doses de $\mathrm{Pb}$ (250, 500 e $1.000 \mathrm{mg} \mathrm{kg}^{-1}$ de solo) em experimento conduzido em vasos com drenagem livre para a água, a céu aberto, por 10 anos. As extrações seqüenciais detectaram diferenças entre as formas de adsorção dos dois metais nos solos. O Cd foi detectado em todas as frações, principalmente na trocável e na orgânica, enquanto que o $\mathrm{Pb}$ se concentrou nas frações orgânica e residual. O Cd apresentou maior mobilidade nos solos, notadamente naqueles com menor teor de argila; a mobilidade do chumbo foi muito baixa.
\end{abstract}

Palavras-chave: metais pesados, extrações químicas seqüenciais, biodisponibilidade.

\section{ABSTRACT}

The hazard environment impact and soil pollution by heavy metals should be evaluated not only by total concentration but mainly by their bioavailability because this property is better related with plant uptake. The objective of this experiment was to evaluate, trough sequential chemical extractions, the bioavailability of cadmium and lead in six soils of Rio Grande do Sul State, Brazil. The soil samples investigated come from a long term experiment in which 10 years ago three levels of $\mathrm{Cd}$ and $\mathrm{Pb}$ were applied $(1.25,2.50$ and 5.0mg Cd and 250, 500, 1000mg Pb kg-1 soil). The sequential extractions detected differences among the adsorption forms of the two metals in the soils. The Cd was detected in all fractions, mainly in the exchangeable and organic, while $\mathrm{Pb}$ concentrated on the organic and residual fractions. The Cd presented larger mobility in soils, especially in those with smaller clay content; mobility of lead was very low in all soils.

Key words: heavy metals, sequential chemical extractions, bioavailability.

\section{INTRODUÇÃO}

O teor total de metais no solo geralmente não é um índice adequado para estimar a biodisponibilidade dos mesmos em solos contaminados, pois somente parte apresenta mobilidade no perfil do solo e está disponível para a absorção pelas raízes das plantas. Geralmente, as formas solúveis em água e trocáveis são consideradas prontamente móveis e disponíveis para as plantas. Os metais adsorvidos nas entrecamadas de argilas, precipitados com carbonatos, ligados a óxidos ou complexados pela matéria orgânica podem ser considerados menos biodisponíveis, dependendo da combinação das propriedades químicas e físicas do solo (SPOSITO et al., 1982).

IPrograma de Pós-graduação em Ciência do Solo, Universidade Federal do Rio Grande do Sul (UFRGS). Porto Alegre, RS, Brasil. Email: clauncosta@yahoo.com.br.

IDepartamento de Solos, UFRGS, CP 15.100, 90001-970, Porto Alegre, RS, Brasil. E-mail: egon.meurer@ufrgs.br. *Autor para correspondência. 
Como os metais pesados se encontram no solo sob diferentes formas químicas, têm sido propostas extrações seqüenciais com extratores com agressividades crescentes (TESSIER et al., 1979) para caracterizar estas formas (GOMES, 1996). Vários métodos têm sido empregados em extrações seqüenciais de metais pesados. Eles variam no número de frações extraídas e na ordem e tipo de extratores utilizados. O processo de fracionamento começa com o extrator mais fraco e termina com o mais forte, separando entre cinco a sete frações (KABALA \& SINGH, 2001). A fração solúvel/trocável, presente na maioria dos procedimentos de extração seqüencial, é considerada a mais móvel e biodisponível (SASTRE et al., 2001). Estudos de fracionamento de metais pesados realizados por GOMES (1996) indicaram a importância dos óxidos de $\mathrm{Fe}$ e $\mathrm{Al}$ na retenção de metais, por meio de mecanismos de adsorção específica e/ou coprecipitação. GAIVIZZO (2001) estudou os efeitos da aplicação de lodo petroquímico, durante seis anos, sobre a mobilidade de $\mathrm{Cu}, \mathrm{Pb}, \mathrm{Ni}, \mathrm{Zn}$ e $\mathrm{Cd}$ em um Argissolo Vermelho-amarelo eutrófico, e a sua relação com as formas químicas dos metais no solo nove anos após a última aplicação. As extrações seqüenciais revelaram maiores quantidades de $\mathrm{Cu}$ e $\mathrm{Pb}$ nas frações orgânica e residual; o $\mathrm{Ni}$ e o $\mathrm{Zn}$ ocorreram principalmente na fração mineral, enquanto que o $\mathrm{Cd}$ distribuiu-se eqüitativamente entre a fração mineral e a orgânica. AMARAL SOBRINHO et al. (1997) incubaram amostras do horizonte Bt de um Podzólico Vermelho-Amarelo com resíduo siderúrgico durante 18 meses. Os autores observaram decréscimo na solubilidade de todos os metais analisados (Zn, Mn, $\mathrm{Pb}, \mathrm{Ni}$, Cd e Cu) através de extração seqüencial. Com o fracionamento, foi possível concluir que esse decréscimo estava relacionado à passagem da forma trocável àquelas menos móveis, ligadas a óxidos e residual.

Esta pesquisa fundamenta-se na observação de que muitos estudos com extrações químicas seqüenciais são feitos em solos submetidos a curtos períodos de incubação com os metais, o que pode muitas vezes ser insuficiente para predizer a dinâmica de suas reações, pois o solo é um sistema dinâmico e aberto. Este estudo teve por objetivo avaliar, mediante extrações químicas seqüenciais, a biodisponibilidade de cádmio e de chumbo em amostras de seis solos do Estado do Rio Grande do Sul, nos quais os metais foram adicionados há 10 anos.

\section{MATERIAL E MÉTODOS}

Este trabalho foi realizado com amostras de seis solos, coletadas de um experimento instalado em
1994, e conduzido a céu aberto em área cercada do Departamento de Solos da Faculdade de Agronomia da Universidade Federal do Rio Grande do Sul (UFRGS). Originalmente os solos haviam sido coletados no horizonte superficial (0 a $20 \mathrm{~cm}$ ) em áreas com vegetação nativa. A classificação taxonômica dos solos e alguns atributos físicos e químicos são apresentados na tabela 1. Em 1994, foram aplicadas nas amostras desses solos por MISSIO (1996) as seguintes quantidades de cádmio e chumbo (nas formas de $\mathrm{CdCl}_{2} \cdot \mathrm{H}_{2} \mathrm{O}$ e $\mathrm{PbCl}_{2}$, respectivamente), definidas de acordo com o teor de argila: no PVd1, com teor de argila menor do que $200 \mathrm{~g} \mathrm{~kg}^{-1}$, 1,5mg de Cd e 250mg de $\mathrm{Pb} \mathrm{kg}^{-1}$ de solo; nos solos NVdf1, CHa2 e LVd3, com teores de argila entre 200 a $400 \mathrm{~g} \mathrm{~kg}^{-1}$, 2,5mg de Cd e 500mg de $\mathrm{Pb}$ $\mathrm{kg}^{-1}$ de solo e nos solos CHa1 e LVdf1, com teores de argila maiores do que $400 \mathrm{~g} \mathrm{~kg}^{-1}$, 5,0mg de Cd e 1.000mg de $\mathrm{Pb} \mathrm{kg}^{-1}$ de solo. Após a adição das doses de Cd e de $\mathrm{Pb}$, as amostras dos solos foram homogeneizadas e acondicionadas em sacos plásticos por 50 dias, com teor de umidade igual a 75\% da capacidade de campo. Após este período, as amostras foram transferidas para as unidades experimentais, compostas por vasos de PVC (250mm de diâmetro), com orifício na parte inferior para drenagem livre da água percolada. Nos vasos, acomodaram-se duas camadas de solo: uma superficial (12 litros), na qual foram adicionados o $\mathrm{Cd}$ e o $\mathrm{Pb}$, e uma subsuperficial (oito litros), sem tratamento, separada da primeira por meio de uma tela de polietileno com dois milímetros de abertura de malha. Em janeiro de 2003, a camada superficial dos vasos foi retirada e homogeneizada, sendo coletada uma amostra de cada solo, para a as extrações seqüenciais (100g) e para a extração da solução dos solos (600g). A amostragem da camada subsuperficial, por estar compactada, foi feita com trado calador, sendo retiradas oito subamostras por vaso. Todas as amostras foram secadas em estufa a $40^{\circ} \mathrm{C}$ até peso constante, moídas em almofariz de porcelana, e tamisadas em peneira com orifícios de $2 \mathrm{~mm}$ de diâmetro.

\section{Extração seqüencial dos metais}

O fracionamento e a quantificação das formas químicas de $\mathrm{Cd}$ e de $\mathrm{Pb}$ (extração seqüencial) nas amostras do solo seguiram a metodologia descrita em GAIVIZZO (2001). A fração solúvel em água (F1) foi extraída com $\mathrm{H}_{2} \mathrm{O}$ destilada-deionizada; a fração trocável (F2), com $\mathrm{Mg}\left(\mathrm{NO}_{3}\right)_{2} 0,1 \mathrm{~mol} \mathrm{~L}^{-1}$; a fração orgânica (F3), com DTPA 5 x $10^{-3} \mathrm{~mol} \mathrm{~L}^{-1}$ a pH 5,3; a fração orgânica (F4), com NaOH 0,5 $\mathrm{mol} \mathrm{L}^{-1}$ e a fração residual (F5) com $\mathrm{HNO}_{3}-\mathrm{H}_{2} \mathrm{O}_{2}$. Os teores totais de Cd e de $\mathrm{Pb}$ nas amostras foram obtidos por extração com $\mathrm{HNO}_{3}-\mathrm{H}_{2} \mathrm{O}_{2}$, conforme metodologia da USEPA(1986), 
Tabela 1 - Alguns atributos originais dos solos utilizados no experimento (MISSIO, 1996).

\begin{tabular}{|c|c|c|c|c|c|}
\hline \multirow{2}{*}{ Classificação taxonômica } & $\mathrm{pH}^{(1)}$ & $\mathrm{Fe}^{(2)}$ & $\mathrm{CTC}^{(3)}$ & Matéria orgânica & Argila \\
\hline & & $\mathrm{g} \mathrm{kg}^{-1}$ & $\mathrm{cmol}_{\mathrm{c}} \mathrm{dm}^{-3}$ & ---------- g kg & ------ \\
\hline Argissolo Vermelho distrófico arênico - PVd1 & 4,8 & 0,04 & 1,68 & 10 & 120 \\
\hline Nitossolo Vermelho distroférrico latossólico - NVdf1 & 5,1 & 0,43 & 3,67 & 33 & 360 \\
\hline Cambissolo Húmico alumínico típico - CHa2 & 4,7 & 0,35 & 2,31 & 40 & 460 \\
\hline Latossolo Vermelho distrófico típico - LVd3 & 4,8 & 0,26 & 3,89 & 39 & 300 \\
\hline Cambissolo Húmico alumínico típico - CHa1 & 4,2 & 1,39 & 5,46 & 75 & 480 \\
\hline Latossolo Vermelho distroférrico típico - LVdf1 & 4,5 & 0,64 & 6,74 & 40 & 520 \\
\hline
\end{tabular}

${ }^{(1)}$ relação solo:água destilada 1:1; ${ }^{(2)}$ Fe extraído por oxalato de amônio; ${ }^{(3)}$ CTC a pH 7.

Método 3050. Detalhes adicionais dos procedimentos encontram-se em COSTA(2005). O Cd foi determinado em espectrofotômetro de absorção atômica em forno de grafite Perkin Elmer modelo Analyst 100 com amostrador automático AS 72 e o Pb por espectofotometria de absorção atômica (Perkin Elmer, modelo 3110) por atomização em chama de ar-acetileno.

Mobilidade dos metais

Para estimar a mobilidade do $\mathrm{Cd}$ e do $\mathrm{Pb}$ no solo, foi empregado o índice "fator de mobilidade (FM)" proposto por KABALA \& SINGH (2001). Este índice é obtido a partir da fórmula: $\mathrm{FM}=\left[\left(\mathrm{F}_{1}+\mathrm{F}_{2}\right) /\right.$ $\left.\left(\mathrm{F}_{1}+\mathrm{F}_{2}+\mathrm{F} 3+\mathrm{F}_{4}+\mathrm{F}_{5}\right)\right] \times 100$, em que $\mathrm{F}$ com os índices $1,2,3,4$,e 5 representam as frações da extração seqüencial discriminadas anteriormente.

\section{RESULTADOS E DISCUSSÃO}

Teores de cádmio e de chumbo nas frações da extração seqüencial

A quantidade total obtida pela soma das concentrações encontradas em cada fração foi menor ou maior do que o total obtido com uma extração única com $\mathrm{HNO}_{3}-\mathrm{H}_{2} \mathrm{O}_{2}$ (Tabela 2). Isso se deve, provavelmente, às perdas de material durante os processos de extração seqüencial e/ou à falta de especificidade dos extratores. Por esse motivo, conforme recomendação de MCLAREN \& CRAWFORD (1973), a adequação da metodologia de extração deve ser testada por análise de regressão entre o teor total determinado pela digestão com $\mathrm{HNO}_{3}-\mathrm{H}_{2} \mathrm{O}_{2}$ e a soma dos teores obtidos nas frações. Os resultados mostraram (Tabela 2) que, mesmo diferindo em valores absolutos, o somatório das quantidades dos metais determinadas nas frações das extrações seqüenciais apresentou alta relação com os teores totais determinados pela digestão única com o $\mathrm{HNO}_{3}-\mathrm{H}_{2} \mathrm{O}_{2}$ $\left(\mathrm{Y}=-124,4+0,99 \mathrm{X}\right.$ com $\mathrm{R}^{2}=0,93 * *$ para o $\mathrm{Cd}$ e $\mathrm{Y}=$ $0,56+1,0039 \mathrm{X}$ com $\mathrm{R}^{2}=0,99 * *$ para o $\mathrm{Pb}$ ). Assim, mesmo que os resultados do fracionamento não correspondam exatamente aos valores "reais" dos metais nas diferentes frações, estes podem apresentar coerência em termos relativos, podendo ser considerados satisfatórios, tendo em vista os possíveis erros a que esta metodologia está sujeita. Porém, a regressão e o

Tabela 2 - Teores de cádmio nas frações da extração seqüencial, distribuição porcentual, somatório das frações e teor total nas amostras da camada superficial $(0-20 \mathrm{~cm})$ dos $\operatorname{solos}^{1}$

\begin{tabular}{|c|c|c|c|c|c|c|c|c|c|c|c|c|c|c|}
\hline \multirow{3}{*}{$\begin{array}{l}\text { Solo } \\
\text { PVd1 }\end{array}$} & \multicolumn{2}{|c|}{$\mathrm{H}_{2} \mathrm{O}$} & \multicolumn{2}{|c|}{$\left(\mathrm{MgNO}_{3}\right)_{2}$} & \multicolumn{2}{|c|}{ DTPA } & \multicolumn{2}{|c|}{$\mathrm{NaOH}$} & \multicolumn{2}{|c|}{$\mathrm{HNO}_{3}-\mathrm{H}_{2} \mathrm{O}$} & \multicolumn{2}{|c|}{$\begin{array}{l}\text { Somatório das } \\
\text { frações }\end{array}$} & \multicolumn{2}{|c|}{$\begin{array}{c}\text { Total }^{2} \\
\left(\mathrm{HNO}_{3}-\mathrm{H}_{2} \mathrm{O}\right)\end{array}$} \\
\hline & & & & & & & & $\mathrm{mg} \mathrm{kg}^{-1}$ & & & & & & \\
\hline & 0,045 & $(8,2)$ & 0,2 & $(36,4)$ & 0,02 & $(3,6)$ & 0,085 & $(15,5)$ & 0,1 & $(18,2)$ & 0,45 & $(81,8)$ & 0,55 & (100) \\
\hline NVdf1 & 0,25 & $(13,2)$ & 0,72 & $(37,9)$ & 0,05 & $(2,6)$ & 0,1 & $(5,3)$ & 0,73 & $(38,4)$ & 2,30 & $(121,0)$ & 1,90 & (100) \\
\hline СHa2 & 0,23 & $(7,2)$ & 0,65 & $(20,3)$ & 0,67 & $(20,9)$ & 0,79 & $(24,7)$ & 0,56 & $(17,5)$ & 2,90 & $(90,6$ & 3,20 & (100) \\
\hline LVd3 & 0,03 & $(0,9)$ & 1,2 & $(37,5)$ & 0,95 & $(29,7)$ & 0,87 & $(27,2)$ & 0,95 & $(29,7)$ & 4,00 & $(125,0)$ & 3,20 & (100) \\
\hline CHa1 & 0,09 & $(1,7)$ & 1,35 & $(25,5)$ & 1,44 & $(27,2)$ & 0,67 & $(12,6)$ & 0,95 & $(18,0)$ & 4,50 & $(84,9)$ & 5,30 & (100) \\
\hline LVdf1 & 0,18 & $(3,0)$ & 0,9 & $(15,2)$ & 0,9 & $(15,2)$ & 1,32 & $(22,4)$ & 2,70 & $(45,8)$ & 6,00 & $(101,7)$ & 5,90 & (100) \\
\hline
\end{tabular}

${ }^{1}$ Para cada fração, a primeira coluna refere-se ao teor ( $\left.\mathrm{mg} \mathrm{kg}^{-1}\right)$ e a segunda à contribuição percentual (\%) em relação ao total extraído com o $\left(\mathrm{HNO}_{3}-\mathrm{H}_{2} \mathrm{O}\right)$.

${ }^{2}$ Teor total de Cd em extração única com $\mathrm{HNO}_{3}-\mathrm{H}_{2} \mathrm{O}$, conforme descrito em USEPA (1986).

Ciência Rural, v.37, n.5, set-out, 2007. 
coeficiente de determinação para o cádmio sugerem que, para este metal, os extratores, ou parte deles, utilizados na extração seqüencial não seriam os mais adequados. É possível, também, que o fato esteja relacionado à imprecisão das leituras, devido ao cádmio ser o metal que se encontrava em menores concentrações (ppb) nas frações solúvel e trocável.

A extração seqüencial detectou diferenças entre os solos e a distribuição do cádmio e do chumbo nas diferentes frações e provavelmente acompanhou as suas afinidades de adsorção. Ambos os metais foram detectados em todas as frações, nos seis solos, em quantidades diferenciadas (Tabela 2). As frações solúvel em água e trocável representaram 18\% (no LVdf1) a 51\% (no NVdf1) do total do Cd e menos do que $3 \%$ (no NVdf1) do total do Pb extraído dos seis solos. O Cd retido nas frações orgânicas (DTPA e $\mathrm{NaOH})$ esteve entre 19 (no PVd1) a 56,8\% (LVd3) do total extraído; para o $\mathrm{Pb}$, a concentração variou de 13,6 (PVd1) a 50,8\% (CHa1). Na fração residual, foi detectada a presença de ambos os metais (Tabelas 2 e 3).

O Cd apresentou maior percentual nas frações solúvel em água e trocável, indicando, possivelmente, a maior participação de ligações menos energéticas (eletrostáticas) e, conseqüentemente, possibilitando uma maior mobilidade do metal no solo. No entanto, este metal foi também bastante retido nas frações orgânicas e residuais em todos os solos, indicando que, em condições de baixa competição com outros cátions, ele também tem tendência de permanecer sob formas menos disponíveis, permanecendo adsorvido especificamente à matéria orgânica, a minerais silicatados e a óxidos. Na fração residual do LVdf1, que predominou sobre as demais frações neste solo, pode-se verificar a forte afinidade do cádmio pelos óxidos de ferro (hematita) constituintes dessa classe de solo (Figura 1). Em um estudo com dois solos (Latossolo Vermelho Amarelo e Argissolo Vermelho Amarelo) tratados com lodos de esgoto enriquecidos com metais, OLIVEIRA et al. (2003) observaram, pelas extrações seqüenciais, que compostos orgânicos solúveis formaram complexos especialmente com cádmio e zinco, mantendo elevados os teores destes elementos na fração solúvel em água durante todo o período experimental. O`CONNOR et al. (1984) verificaram que dois mecanismos foram responsáveis pela retenção do Cd nos solos: o primeiro foi a adsorção específica, quando foram adicionadas baixas concentrações do metal $\left(0,01\right.$ a $\left.10 \mathrm{mg} \mathrm{L}^{-1}\right)$; com maiores adições do elemento (100 a $1.000 \mathrm{mg} \mathrm{L}^{-1}$ ), predominaram as reações de troca (ligações eletrostáticas).

O chumbo predominou nas frações orgânica e residual em todos os solos, concentrando-se principalmente na fração residual. Essa distribuição confirma o comportamento típico deste elemento em solos contaminados, ou seja, apresenta alta retenção, baixa mobilidade e biodisponibilidade (ALLOWAY, 1995). Por esse motivo, o chumbo é considerado um dos metais menos móveis no solo, podendo ser complexado pela matéria orgânica, quimiossorvido em óxidos e minerais silicatados e precipitado como carbonato, hidróxido ou fosfato em condições de alto pH (MCBRIDE, 1994). Neste estudo, isso pôde ser evidenciado pelas pequenas porcentagens do elemento nas frações solúvel em água e trocável em todos os tratamentos, indicando pequena contribuição da adsorção não-específica (eletrostática) para este elemento. ARAÚJO et al. (2002) verificaram em 12 classes de solos brasileiros que o chumbo apresentou maior afinidade pelo grupo funcional $\mathrm{OH}$ presente na superfície de caulinita, óxidos e hidróxidos de ferro e alumínio e menor afinidade pelos grupos funcionais das substâncias húmicas. Esta última observação pode

Tabela 3 - Teores de chumbo nas frações da extração seqüencial, distribuição porcentual, somatório das frações e teor total nas amostras da camada superficial $(0-20 \mathrm{~cm})$ dos solos ${ }^{1}$.

\begin{tabular}{|c|c|c|c|c|c|c|c|c|c|c|c|c|c|c|}
\hline \multirow[t]{2}{*}{ Solo } & \multicolumn{2}{|c|}{$\mathrm{H}_{2} \mathrm{O}$} & \multicolumn{2}{|c|}{$\left(\mathrm{MgNO}_{3}\right)_{2}$} & \multicolumn{2}{|c|}{ DTPA } & \multicolumn{2}{|c|}{$\mathrm{NaOH}$} & \multicolumn{2}{|c|}{$\mathrm{HNO}_{3}-\mathrm{H}_{2} \mathrm{O}$} & \multicolumn{2}{|c|}{$\begin{array}{l}\text { Somatório das } \\
\text { frações }\end{array}$} & \multicolumn{2}{|c|}{$\begin{array}{c}\text { Total }^{2} \\
\left(\mathrm{HNO}_{3}-\mathrm{H}_{2} \mathrm{O}\right)\end{array}$} \\
\hline & & & & & & .....m & $\mathrm{kg}^{-1}$. & & & & & & & \\
\hline PVd1 & 3,2 & $(1,9)$ & 1,5 & $(0,9)$ & 11,8 & $(7,1)$ & 10,8 & $(6,5)$ & 140,0 & $(84,3)$ & 167,3 & $(100,8)$ & 166 & (100) \\
\hline NVdf1 & 2,1 & $(0,5)$ & 8,1 & $(2,0)$ & 109,0 & $(26,9)$ & 2,1 & $(0,5)$ & 280,0 & $(69,1)$ & 401,3 & $(98,9)$ & 405,5 & (100) \\
\hline СHa2 & 1,5 & $(0,3)$ & 3,8 & $(0,8)$ & 190,7 & $(40,7)$ & 2,1 & $(0,4)$ & 277,3 & $(59,1)$ & 475,4 & $(101,4)$ & 469 & (100) \\
\hline LVd3 & 2,1 & $(0,4)$ & 0,05 & $(0,1)$ & 97,8 & $(20,4)$ & 5,2 & $(1,1)$ & 371,2 & $(77,5)$ & 476,4 & $(99,5)$ & 479 & (100) \\
\hline CHa1 & 3,1 & $(0,3)$ & 4,1 & $(0,4)$ & 472,2 & $(46,2)$ & 48,0 & $(4,7)$ & 490,7 & $(48,0)$ & $1.018,1$ & $(99,5)$ & 1.023 & (100) \\
\hline LVdf1 & 9,0 & $(0,9)$ & 2,9 & $(0,3)$ & 204,6 & $(20,0)$ & 26,7 & $(2,9)$ & 770,0 & $(75,3)$ & $1.013,2$ & $(99,0)$ & 1.023 & (100) \\
\hline
\end{tabular}

${ }^{1}$ Para cada fração, a primeira coluna refere-se ao teor $\left(\mathrm{mg} \mathrm{kg}^{-1}\right)$ e a segunda à contribuição percentual (\%), em relação ao total extraído com o $\left(\mathrm{HNO}_{3}-\mathrm{H}_{2} \mathrm{O}\right)$.

${ }^{2}$ Teor total de $\mathrm{Cd}$ em extração única com $\mathrm{HNO}_{3}-\mathrm{H}_{2} \mathrm{O}$, conforme descrito em USEPA (1986). 


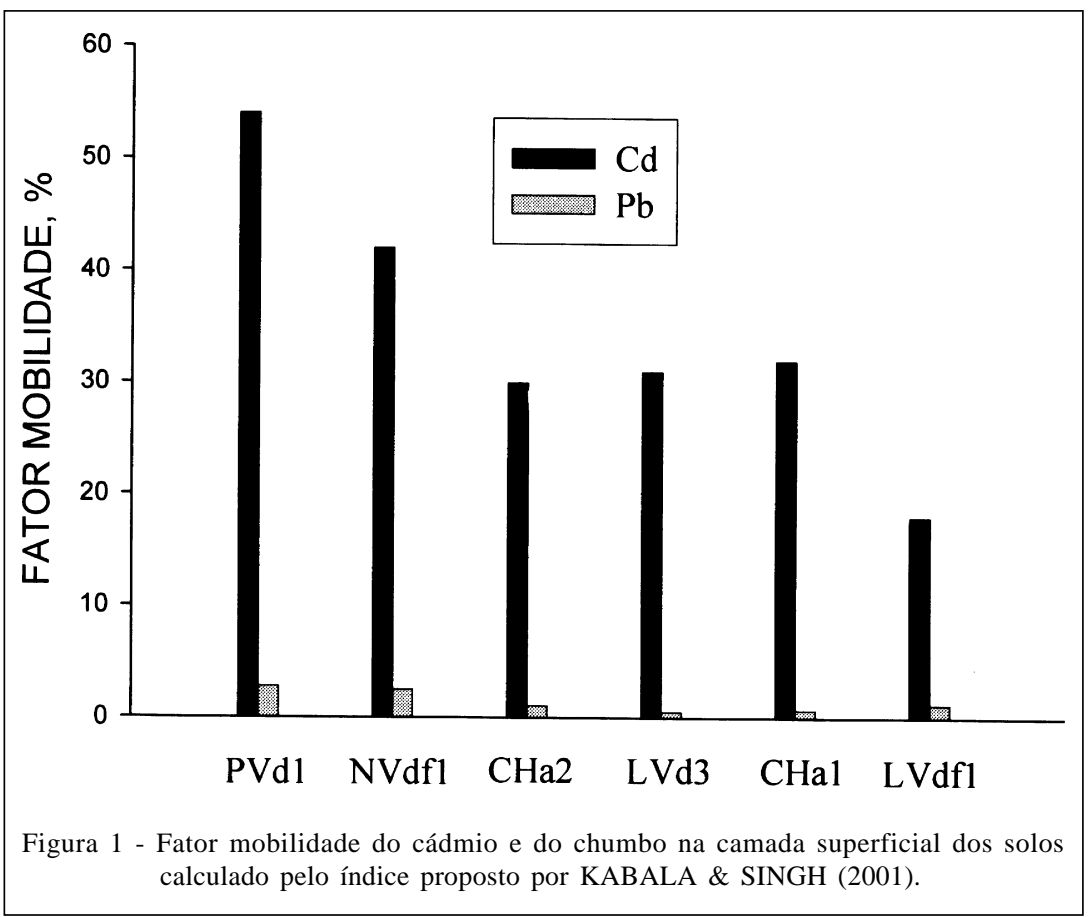

explicar as pequenas quantidades do elemento extraídas com o extrator $\mathrm{NaOH}$ (fração orgânica). Este extrator provoca o rompimento das pontes de $\mathrm{H}+$ das moléculas orgânicas entre si e delas com a superfície do mineral e promove reações de troca de ligantes entre as hidroxilas do meio e os grupos carboxílicos e fenólicos das moléculas de substâncias húmicas pelos quais a mesma se encontra coordenada na superfície hidroxilada do mineral (STEVENSON, 1995). Dessa forma, o $\mathrm{NaOH}$ extrai as substâncias húmicas do solo e, conseqüentemente, os metais a elas complexados. Neste sentido, uma outra hipótese para explicar o predomínio de chumbo na fração residual é que, apesar da utilização de dois extratores (DTPA e $\mathrm{NaOH}$ ) para extrair as frações orgânicas, estes não tenham sido suficientemente eficientes, sobrando ainda material orgânico altamente estável para ser solubilizado pela digestão com $\mathrm{HNO}_{3}-\mathrm{H}_{2} \mathrm{O}_{2}$.

Mobilidade do Cd e do Pb nos solos

Considerando que a ocorrência dos metais nas frações solúvel em água e trocável pode ser um indicativo de biodisponibilidade, aplicou-se aos dados obtidos o índice "fator de mobilidade” utilizado por KABALA \& SINGH (2001) para facilitar a comparação entre metais em solos com atributos físicos e químicos diferentes. Altos valores para o fator de mobilidade têm sido interpretados como indicativos de alta biodisponibilidade de metais pesados em solos (MA
\& RAO, 1997). Em todos os solos, a mobilidade do Cd foi maior que a do $\mathrm{Pb}$ (Figura 1).

Constata-se que os maiores valores do fator de mobilidade para os dois metais ocorreram no PVd1 e no NVdf1. O solo PVd1 (unidade regional Bom Retiro) caracteriza-se por apresentar maiores teores de areia (quartzo) que os demais, fração argila com baixa CTC e, conseqüentemente, baixa fertilidade natural (distrófico). Da mesma forma, o solo NVdf1 (unidade regional Estação) também tem baixa CTC, pelo fato de apresentar predomínio de caulinita e óxidos de ferro (PCZ de 7,8 9,5) na fração argila. Esses dois solos apresentam, igualmente, menores teores de matéria orgânica que os demais (Tabela 1) e, assim, menor número de grupos funcionas de superfície. Esses resultados evidenciam a importância da matéria orgânica, dos óxidos e dos minerais silicatados com alta CTC na adsorção de metais no solo. Resultados semelhantes foram encontrados por FORBES et al. (1976), em relação à afinidade dos metais pela goethita $(\mathrm{Pb}>\mathrm{Cd})$, e por BENJAMIN \& LECKIE (1981), para os óxidos de ferro em geral $(\mathrm{Pb}>\mathrm{Cd})$. Assim, os óxidos de ferro, juntamente com a matéria orgânica, parecem ser os constituintes mais importantes do solo para reações de adsorção específica, o que possivelmente ocorreu com mais intensidade nos solos NVdf1, LVd3 e LVdf1. A eletronegatividade é um parâmetro que também pode indicar os metais que podem ser adsorvidos com maior preferência nos minerais silicatados e nos óxidos: metais 
mais eletronegativos poderão formar ligações covalentes mais fortes com átomos de oxigênio nas superfícies dos minerais.

\section{CONCLUSÕES}

As extrações seqüenciais detectaram diferenças entre as formas de adsorção dos dois metais adicionados nos seis solos há 10 anos. O Cd foi detectado em todas as frações, principalmente na trocável e na orgânica, enquanto que o Pb concentrouse nas frações orgânica e residual. O “fator mobilidade” indicou que o $\mathrm{Cd}$ apresentou maior mobilidade nos solos, podendo apresentar maior risco ambiental, principalmente em solos com pouca matéria orgânica, baixos teores de óxidos de ferro e com baixa CTC. A mobilidade do chumbo foi muito baixa nos solos, indicando que este metal pode apresentar menor risco de contaminação do ambiente.

\section{AGRADECIMENTOS}

Os autores agradecem à Fundação de Amparo à Pesquisa do Estado do Rio Grande do Sul (FAPERGS) e ao Conselho Nacional de Desenvolvimento Científico e Tecnológico (CNPq) pelo apoio financeiro ao trabalho.

\section{REFERÊNCIAS}

ALLOWAY, B.J. Heavy metals in soils. 2.ed. Glasgow: Blackie Academic, 1995. 364p.

AMARAL SOBRINHO, N.M.B et al. Solubilidade de metais pesados em solo tratado com resíduo siderúrgico. Revista Brasileira de Ciência do Solo, v.21, p.9-16, 1997.

ARAÚJO, W.S. et al. Relação entre adsorção de metais pesados e atributos químicos e físicos de classes de solos do Brasil. Revista Brasileira de Ciência do Solo, v.26, p.17-27, 2002.

BENJAMIN, M.M.; LECKIE, J.O. Multiple-site adsorption of $\mathrm{Cd}, \mathrm{Zn}$ and $\mathrm{Pb}$ on amorphous iron oxyhydroxides. Journal Colloid Interface Science, v.79, p.209-221, 1981.

COSTA, C.N. Biodisponibilidade de metais pesados em solos do Rio Grande do Sul. 2005. 110f. Tese (Doutorado em Ciência do Solo) - Programa de Pós-graduação em Ciência do Solo, Universidade Federal do Rio Grande do Sul, Porto Alegre.

FORBES, E.A. et al. The specific adsorption of divalent Cd, $\mathrm{Co}, \mathrm{Pb}$ and $\mathrm{Zn}$ on goethite. Journal of Soil Science, v.27, p.154-166, 1976.
GAIVIZZO, L.H.B. Fracionamento e mobilidade de metais pesados em solo com descarte de lodo industrial. 2001. 123f. Tese (Doutorado em Ciência do Solo) - Programa de Pós-graduação em Ciência do Solo, Faculdade de Agronomia, Universidade Federal do Rio Grande do Sul, Porto Alegre.

GOMES, P.C. Fracionamento e biodisponibilidade de metais pesados por calagem e concentrações de metais em Latossolo Vermelho-amarelo. 1996. 161f. Tese (Doutorado em Solos) - Universidade Federal de Viçosa

KABALA, C.; SINGH, B.R. Fractionation and mobility of copper, lead, and zinc in soil profiles in the vicinity of a copper smelter. Journal of Environmental Quality, v.30, p.485492, 2001.

MA, L.Q.; RAO, G.N. Chemical fractionation of cadmium, copper, nickel and zinc in contaminated soils. Journal of Environmental Quality, v.26, p.259-264, 1997.

MISSIO, E. Avaliação da disponibilidade de alguns metais pesados para as plantas. 1996. 120f. Dissertação (Mestrado em Ciência do Solo) - Programa de Pós-graduação em Ciência do Solo, Faculdade de Agronomia, Universidade Federal do Rio Grande do Sul, Porto Alegre.

McBRIDE, M.B. Environmental chemistry of soils. New York: Oxford University, 1994. 406p.

McLAREN, R.G.; CRAWFORD, D.V. Studies on soil copper. I. The fractionation of copper in soils. Journal of Soil Science, v.24, n.2, p.172-181, 1973.

O`CONNOR, G.A. et al. Sorption of cadmium by calcareous soils: influence of solution composition. Soil Science Society of America Journal, v.48, p.1244-1247, 1984.

OLIVEIRA, C. et al. Solubilidade de metais pesados em solos tratados com lodo de esgoto enriquecido. Revista Brasileira de Ciência do Solo, v.27, p.171-181, 2003.

SASTRE, I. et al. Behavior of cadmium and nickel in a soil amended with sewage sludge. Land degradation and development, v.12, p.27-33, 2001.

SPOSITO, G. et al. Trace metal chemistry in arid zone field soils amended with sewage sludge: fractionation of $\mathrm{Ni}, \mathrm{Cu}, \mathrm{Zn}$, $\mathrm{Cd}$ and $\mathrm{Pb}$ in solid phases. Soil Science Society America Journal, v.46, p.260-264, 1982.

STEVENSON, F.J. Humus chemistry. Genesis, composition, reactions. 2.ed. New York: Jonh Wiley, 1995. 496p.

USEPA-UNITED STATES ENVIRONMENTAL PROTECTION AGENCY. Test methods for evaluating solid wastes. Physical/Chemical Methods - SW 846. Washington, DC, 1986. 3500p.

TESSIER, R.W. et al. Sequential extraction procedure for the speciation of particulate trace-elements. Environmental Technology, v.15, p.844-851, 1979. 